\title{
User Association in Massive MIMO and mmWave Enabled HetNets Powered by Renewable Energy
}

\author{
Bingyu Xu*, Yue Chen*, Maged Elkashlan*, Tiankui Zhang ${ }^{\dagger}$, and Kai-Kit Wong ${ }^{\ddagger}$ \\ ${ }^{*}$ School of Electronic Engineering and Computer Science, Queen Mary University of London, London, UK \\ ${ }^{\dagger}$ School of Information and Communications Engineering, Beijing University of Posts and Telecommunications, Beijing, China \\ ${ }^{\ddagger}$ Department of Electronic and Electrical Engineering, University College London, London, UK
}

\begin{abstract}
This paper considers a hybrid heterogeneous network (HetNet), where macro cells adopt massive multiple-input multiple-output (MIMO), and small cells adopt millimeter wave (mmWave) transmissions. We assume that all base stations (BSs) are solely powered by the renewable energy. The implementation of these emerging techniques has a substantial effect on the user association (UA). Motivated by this, we formulate a user association problem to maximize the network utility while the power cost of each BS does not exceed the harvested energy. To solve it, a low complexity distributed UA algorithm is proposed. The results demonstrate that the proposed algorithm achieves higher throughput than the max reference signal received power (RSRP) and max signal-to-interference-plus-noise ratio (SINR) UAs. It also shows that increasing the number of antennas at the macro cell BS with more power consumption, the throughput continues to increase by using the proposed algorithm, compared to the decrease in throughput by using the existing ones. Increasing the number of mmWave BSs, mmWave BS antennas or mmWave bandwidths can significantly improve the throughput. Compared with massive MIMO macro cells, mmWave small cells play a dominant role in enhancing the throughput of the networks due to the larger bandwidths.
\end{abstract}

\section{INTRODUCTION}

The fifth generation $(5 \mathrm{G})$ cellular networks will be designed for the provision of the anticipated 1000x data increase [1]. To achieve this target, several emerging techniques such as dense heterogeneous networks (HetNets), massive multi-input multi-output (MIMO), millimeter wave (mmWave), etc. are developed $[2,3]$. However, none of these techniques can solely accomplish $5 \mathrm{G}$ requirements, and the integration of them is identified as a promising solution [2,3].

Massive MIMO and mmWave are presumably two essential enabling $5 \mathrm{G}$ technologies for improving capacity [2]. The rationale behind massive MIMO is that each base station (BS) is equipped with large number of antennas and communicates with multiple terminals over the same time and frequency band [4]. In this way, energy and spectral efficiency can be enhanced with orders of magnitude compared to singleantenna systems [4]. Due to the shortage of low cellular frequency band, mmWave spectrum becomes a promising mobile broadband [5]. In fact, mmWave spectrum has been adopted in the standards such as IEEE 802.11ad [6] for supporting gigabit per second (Gbps) transmission.

A combination of massive MIMO and mmWave is potentially applied in the hybrid HetNets [5], where massive
MIMO transmissions operate in macrocells with low cellular frequencies [7], and mmWave transmissions operate in small cells with high frequencies. In such HetNets, user association (UA) that determines whether a user is associated with a BS will be more complicated $[2,8]$, and current UA designs may not be capable of full exploiting the benefits provided by massive MIMO and mmWave. There are at least two-fold issues to be addressed: 1) Compared to the current narrow cellular bandwidths (BWs), users in the mmWave cells will be allocated gigahertz (GHz) BWs. As such, new UA methods have to account for the impact of diverse system BWs; and 2) the shorter wavelengths of mmWave frequencies enable mmWave BSs to pack more antennas and hence provide larger array gains. The simple UA metric based on the minimumdistance rule [8] may become inefficient, particularly when massive MIMO is applied in the macro cells [7]. The antenna array gains in the mmWave cells will be different from the cellular antenna array gains in the macrocells. As such, new UA schemes should also address the impact of large array gains.

Recent research efforts have been devoted to examine the UA for load balancing in massive MIMO cellular networks [9] and mmWave wireless networks [10]. In [9], small and large massive MIMO BSs were deployed in the cellular networks and max-rate based and load based associations were analyzed. In [10], the problem of UA in a $60-\mathrm{GHz}$ wireless network was considered, and a resource allocation solution was proposed to guarantee the balance and fairness of the system. However, UA in massive MIMO and mmWave networks is still far from being well understood, particularly in the hybrid HetNets where massive MIMO and mmWave transmissions co-exist.

In addition, 5G networks are expected to be more energy efficient, and more base stations with the capability of harvesting renewable energy will be deployed for being greener $[1,3]$. As a result, energy harvesting will play a crucial role in reducing the power consumption and operational expenditure (OPEX) of the networks. However, the amount of energy harvested from the renewable energy sources such as solar panels or wind turbines depends on many factors such as environment and is random [11], which has an effect on the associations between users and renewable energy-powered BSs.

Considering the aforementioned important aspects, this paper studies UA in two-tier hybrid HetNets including massive 


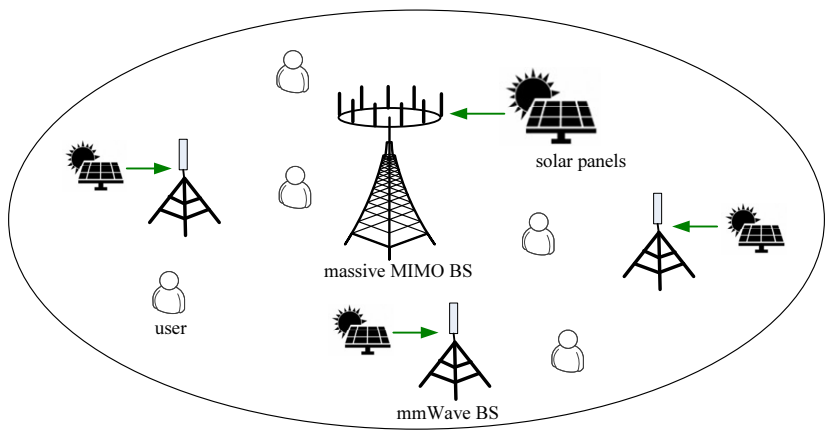

Fig. 1. An example of a two-tier hybrid HetNets powered by the solar panels

MIMO aided macrocells and mmWave small cells, where all BSs are powered by the renewable energy sources. To the best of our knowledge, this field is novel and has not be investigated yet. Due to the high cost of mmWave RF chains and power consumption, the low-cost low-complexity analog beamforming is applied in the mmWave small cells. We address the impacts of different array gains, BWs, and harvested energies on the UA in such hybrid HetNets. In doing so, a UA problem is established to maximize the sum logarithmic throughput under energy constraint. We propose a low-complexity distributed UA algorithm for solving this problem. The results show that the proposed UA algorithm can efficiently integrate the advantages of massive MIMO with mmWave, and achieves higher throughput than the max reference signal received power (RSRP) and max signal-tointerference-plus-noise ratio (SINR) UA schemes.

This paper is organized as follows. Section II presents the system model. Section III formulates the problem, gives the Lagrangian analysis and introduces the distributed UA scheme of our design. The last two sections shows the simulation results and summarizes our conclusions respectively.

\section{SySTEM DESCRIPTIONS}

We focus on a two-tier hybrid heterogeneous network powered by the renewable energy sources such as solar panels and wind turbines, in which the first tier consists of massive multiple-input multiple-output (MIMO) macro cells in the low cellular frequencies (sub-6 GHz), and the second tier consists of the mmWave small cells in the mmWave frequencies, as shown in Fig. 1 . We denote by $\mathcal{U}$ the set of all users, $\mathcal{B}_{\mathrm{c}}$ the set of macro cell BSs, and $\mathcal{B}_{\mathrm{mmW}}$ the set of mmWave small cell BSs. Each marco cell BS has $N_{\mathrm{c}}$ antennas and simultaneously communicates with $S$ single-antenna users over the same time and cellular frequency band $\left(N_{\mathrm{c}} \gg S \geq 1\right)$, while each mmWave small cell BS has $N_{\mathrm{mmW}}$ antennas with a sectored gain pattern and serves one single-antenna user over the same time and mmWave frequency band ${ }^{1}$.

We define the UA indicator $x_{i j}$ to represent if user $j \in \mathcal{U}$

${ }^{1}$ Here, each user is assumed to be equipped with one single omnidirectional antenna [12]. is associated with $\mathrm{BS} i \in\left\{\mathcal{B}_{\mathrm{c}}, \mathcal{B}_{\mathrm{mmW}}\right\}$ as

$$
x_{i j}= \begin{cases}1, & \text { if user } j \text { is associated with BS } i \\ 0, & \text { otherwise }\end{cases}
$$

\section{A. Energy Harvesting}

Each BS harvests the energy from the environment. The energy harvesting process at the BS $i$ follows a stationary stochastic process with the probability density function $f_{i}\left(z_{i}\right)=1 /\left(b_{i}-a_{i}\right), \forall z_{i} \in\left[a_{i}, b_{i}\right]$ where $a_{i}$ and $b_{i}$ are the minimum and maximum harvested energy of BS $i$ respectively [11]. The energy harvesting rate is constant in each time slot and may change from one time slot to another. In practice, the energy harvesting rate may not change within several seconds. We denote $P_{i}^{\mathrm{G}}$ as the green power of the BS $i$ harvested from the renewable energy sources.

\section{B. Downlink Throughput}

1) Massive MIMO Macro Cell: The MBS uses linear zeroforcing beamforming (ZFBF) to transmit $S$ user-streams with equal power assignment. We note that there is no inter-tier interference between the macro cells and mmWave small cells due to the different frequency bands. When a user $j$ is associated with the MBS $i \in \mathcal{B}_{\mathrm{c}}$, the downlink throughput of user $j$ is given by

$$
\tau_{i j}\left(x_{i j}\right)=\left(\sum_{j \in \mathcal{U}} x_{i j}\right)^{-1} S W_{i} \log \left(1+\gamma_{i j}\right),
$$

where

$$
\gamma_{i j}=\left(N_{\mathrm{c}}-S+1\right) \frac{P_{i} g_{i j}}{\sum_{i^{\prime} \neq i} S P_{i^{\prime}} g_{i^{\prime} j}+\Omega_{j} W_{i}},
$$

where $P_{i}$ is the transmit power per user-stream from BS $i$, $W_{i}$ is the bandwidth, $\Omega_{j}$ is the power spectral density of the noise at the user $j$, and $g_{i j}$ is the large-scale fading channel power gain between BS $i$ and user $j$. In (3), $\left(N_{\mathrm{c}}-S+1\right)$ is the array gain achieved by massive MIMO with ZFBF [9].

2) mmWave Small Cell: Each mmWave small cell BS uses analog beamforming with phase shifters. We consider the uniform planar square antenna, and the effective antenna gain for a mmWave $\mathrm{BS} i\left(i \in \mathcal{B}_{\mathrm{mmW}}\right)$ with the beamwidth $\theta=\frac{2 \pi}{\sqrt{N_{\mathrm{mmW}}}}$ is the relative power radiated by BS $i$ in the direction of the user, which is described as a random variable following [13]

$$
G_{i}=\left\{\begin{array}{l}
\frac{N_{\mathrm{mmW}}, \text { with probability } \frac{\theta}{2 \pi}}{\sin ^{2}\left(\frac{3 \pi}{2 \sqrt{N_{\mathrm{mmW}}}}\right)}, \text { with probability }\left(1-\frac{\theta}{2 \pi}\right)
\end{array}\right.
$$

We assume that the antenna gain between the user and its associated BS is the main-lobe gain $N_{\mathrm{mmW}}$. When a user $j$ is associated with the mmWave small cell BS $i \in \mathcal{B}_{\mathrm{mmW}}$, the downlink throughput for user $j$ is given by

$$
\tau_{i j}\left(x_{i j}\right)=\left(\sum_{j \in \mathcal{U}} x_{i j}\right)^{-1} W_{i} \log \left(1+\gamma_{i j}\right),
$$

with

$$
\gamma_{i j}=\frac{P_{i} N_{\mathrm{mmW}} L_{i j}\left(R_{i j}\right)}{\sum_{i^{\prime} \neq i} G_{i^{\prime}} P_{i^{\prime}} L_{i^{\prime} j}\left(R_{i^{\prime} j}\right)+\Omega_{j} W_{i}},
$$


where $L_{i j}\left(R_{i j}\right)$ is the path loss between the user $j$ and its associated mmWave small cell BS $i$ with a distance $R_{i j}$. The path loss laws are different in line-of-sight (LOS) and non-lineof-sight (NLOS) conditions. In this paper, we use the mmWave path loss model proposed in [14], where each mmWave link can be in one of three conditions: LOS, NLOS or outage.

\section{Power Consumption}

The total power consumption for transmitting the user stream to each user associated with the macro cell BS $i \in \mathcal{B}_{\mathrm{c}}$ is given by [15]

$$
P_{i}^{\text {stream }}=P_{i} / \eta_{i}+C_{i}
$$

where $C_{i}=\left(\sum_{m=0}^{3} \Lambda_{m, 0} S^{m}+\sum_{m=0}^{2} \Lambda_{m, 1} S^{m} N_{\mathrm{c}}\right) / S, \eta_{i}$ is the efficiency of power amplifier of BS $i, \Lambda_{m, 0}$ and $\Lambda_{m, 1}$ are the coefficients relying on the power consumption of transceiver chains, coding and decoding, etc. [15], and are detailed in Section IV.

Based on the power consumption models in $[16,17]$, the total power consumption for transmitting the user stream to each user associated with the mmWave small cell BS $i \in$ $\mathcal{B}_{\mathrm{mmW}}$ is given by

$$
P_{i}^{\text {stream }}=P_{i} / \eta_{i}+C_{i}
$$

where $C_{i}=N_{\mathrm{mmW}} P_{\mathrm{ps}}, P_{\mathrm{ps}}$ is the power consumption of the phase shifter.

\section{Problem Formulation And UA Design}

We adopt a proportionally fair network utility optimization approach [18]. The UA problem is that of determining the $x_{i j}$ of each user and BS to maximize the overall network utility while the power consumption of each BS does not exceed its harvested energy, which is expressed as

$$
\begin{aligned}
\max _{\mathbf{X}, \mathbf{K}} & \sum_{j \in \mathcal{U}} \sum_{i \in\left\{\mathcal{B}_{\mathrm{c}}, \mathcal{B}_{\mathrm{mmw}}\right\}} x_{i j} \log \left(\tau_{i j}\left(x_{i j}\right)\right) \\
\text { s.t. } & \sum_{i \in\left\{\mathcal{B}_{\mathrm{c}}, \mathcal{B}_{\mathrm{mmw}}\right\}} x_{i j}=1, \forall j \\
& \sum_{j \in \mathcal{U}} x_{i j}=K_{i}, \forall i \\
& \sum_{j \in \mathcal{U}} x_{i j} P_{i}^{\text {stream }} \leq P_{i}^{\mathrm{G}}, \forall i \\
& x_{i j} \in\{0,1\}, \forall j, \forall i
\end{aligned}
$$

where $\mathbf{X}=\left[x_{i j}\right], \mathbf{K}=\left[K_{i}\right]$. Here, constraints (9b) and (9e) ensure that one user can only be associated with one BS at any time, constraint (9d) indicates that the number of users associated with one BS is restricted by the amount of harvested power. $K_{i}$ in (9c) is the effective load of each BS.

The formulated problem (9) is a combinatorial NP-hard problem due to the discrete nature of the problem. To solve it, we first present the dual analysis.

\section{A. Lagrangian Dual Analysis}

In this subsection, we study the dual problem of (9). The Lagrangian function can be written as

$$
\begin{aligned}
& L(\mathbf{X}, \mathbf{K}, \boldsymbol{\mu}, \mathrm{v})=\sum_{j \in \mathcal{U}} \sum_{i \in \mathcal{B}} x_{i j} \log \left(\varepsilon_{i j}\right)-\sum_{i \in \mathcal{B}} K_{i} \log \left(K_{i}\right) \\
& -\sum_{i \in \mathcal{B}} \mu_{i}\left(\sum_{j \in \mathcal{U}} x_{i j}-K_{i}\right)-\sum_{i \in \mathcal{B}} v_{i}\left(K_{i} P_{i}^{\text {stream }}-P_{i}^{\mathrm{G}}\right),
\end{aligned}
$$

where $\mathcal{B}=\left\{\mathcal{B}_{\mathrm{c}}, \mathcal{B}_{\mathrm{mmW}}\right\}, \varepsilon_{i j}=S W_{i} \log \left(1+\gamma_{i j}\right)$ as $i \in$ $\mathcal{B}_{\mathrm{c}}$, and $\varepsilon_{i j}=W_{i} \log \left(1+\gamma_{i j}\right)$ as $i \in \mathcal{B}_{\mathrm{mmW}}, \mu_{i}$ and $v_{i}$ are Lagrange multipliers.

Accordingly, the dual function $g(\cdot)$ is written as

$$
g(\boldsymbol{\mu}, \mathbf{v})=\left\{\begin{array}{l}
\max _{\mathbf{X}, \mathbf{K}} L(\mathbf{X}, \mathbf{K}, \boldsymbol{\mu}, \mathbf{v}) \\
\text { s.t. } \sum_{i \in \mathcal{B}} x_{i j}=1, \\
x_{i j} \in\{0,1\}, \forall j, \forall i
\end{array}\right.
$$

and the dual problem of (9) is the minimum of $g(\cdot)$ with respect to (w.r.t.) the dual variables, i.e.,

$$
\min _{\substack{\boldsymbol{\mu}, \mathbf{v} \\ \mathbf{v} \geq \mathbf{0}}} g(\boldsymbol{\mu}, \mathbf{v}) .
$$

Given the fixed dual variables $\mu_{i}$ and $v_{i}$, the analytical solution for the maximization of the Lagrangian w.r.t. $\mathbf{X}$ can be written as

$$
x_{i j}^{*}=\left\{\begin{array}{ll}
1, & \text { if } \quad i=i^{*} \\
0, & \text { if } i \neq i^{*}
\end{array},\right.
$$

where $i^{*}=\operatorname{argmax}\left(\log \left(\varepsilon_{i j}\right)-\mu_{i}\right)$. Since the Lagrangian function (10) is ${ }^{i}$ a differentiable concave function of $\mathbf{K}$ with $\partial^{2} L / \partial K_{i}^{2}<0, \forall i$, the optimal primal variables w.r.t. $\mathbf{K}$ for maximizing the Lagrangian can be obtained by letting $\partial L / \partial K_{i}=0$, which yields

$$
K_{i}^{*}=e^{\mu_{i}-v_{i} P_{i}^{\text {stream }}-1} .
$$

From (14), we see that $K_{i}$ is a decreasing function of $P_{i}^{\text {stream }}$, which can be explained by the fact that given the harvested power, increasing the transmit power per user stream will decrease the amount of service BS can supply.

By substituting (13) and (14) into (11), a closed-form expression for dual objective is given by

$$
\begin{aligned}
g(\boldsymbol{\mu}, \mathbf{v})= & \sum_{j \in \mathcal{U}} \max _{i}\left(\log \left(\varepsilon_{i j}\right)-\mu_{i}\right) \\
& +\sum_{i \in \mathcal{B}} e^{\mu_{i}-v_{i} P_{i}^{\text {stream }}-1}+\sum_{i \in \mathcal{B}} v_{i} P_{i}^{\mathrm{G}} .
\end{aligned}
$$

Given the fixed $\boldsymbol{\mu},(15)$ is a differentiable convex function of $\mathbf{v}$ with $\partial^{2} g / \partial v_{i}^{2}>0, \forall i$, hence the optimal dual variables $\mathbf{v}$ for minimizing the dual problem can be obtained by setting $\partial g / \partial v_{i}=0$, which is

$$
v_{i}(t+1)=\frac{\mu_{i}(t)-\log \left(\frac{P_{i}^{\mathrm{G}}}{P_{i}^{\text {stream }}}\right)-1}{P_{i}^{\text {stream }}},
$$


where $t$ is the iteration index, in order to show that $v_{i}$ needs to be updated following $\mu_{i}$. Since $g(\boldsymbol{\mu}, \mathbf{v})$ in (15) is not a differentiable function of $\boldsymbol{\mu}$, the subgradient method is employed to update $\mu_{i}$ according to

$$
\mu_{i}(t+1)=\mu_{i}(t)-\delta(t)\left(K_{i}(t)-\sum_{j \in \mathcal{U}} x_{i j}(t)\right),
$$

where $\delta(t)$ is the step size ${ }^{2}$. By using the updated dual variables $\mu_{i}(t)$ and $v_{i}(t)$ based on (17) and (16), the primal variables $x_{i j}(t)$ and $K_{i}(t)$ can be updated based on (13) and (14). Then, we have the following Proposition:

Proposition 1. The proposed subgradient method in (17) converges to the optimal solution of the dual problem in (12).

Proof: Taking the derivative of dual function $g(\cdot)$ w.r.t. $\boldsymbol{\mu}$ yields

$$
\partial g(\boldsymbol{\mu}, \mathbf{v}) / \partial \mu_{i}=K_{i}\left(\mu_{i}, v_{i}\right)-\sum_{j \in \mathcal{U}} x_{i j}\left(\mu_{i}\right) .
$$

In our primal problem, $K_{i}=\sum_{j \in \mathcal{U}} x_{i j} \leqslant \frac{P_{\mathrm{i}}^{\mathrm{G}}}{P_{i}^{\text {stream }}}$, hence $K_{i}\left(\mu_{i}, v_{i}\right)$ is bounded. As $x_{i j} \in\{0,1\}, \sum_{j \in \mathcal{U}} x_{i j}\left(\mu_{i}\right)$ is also bounded. As such, the subgradient of dual objective is bounded as

$$
\sup _{t}\left\{\left\|\partial g(\boldsymbol{\mu}, \mathbf{v}) / \partial \mu_{i}\right\|\right\} \leq \xi
$$

where $\xi$ is a scalar. Therefore, the necessary condition of the convergence proof holds [19], which confirms Proposition 1.

Because the dual problem is convex, the globally optimal solution for dual problem exists and can be obtained by using the subgradient method. The primal variable $\mathbf{X}$ can be recovered by substituting the optimal dual variables into (13). We note that due to the discrete nature of the primal problem in (9), solving dual problem may not be the same as solving the primal problem. Nevertheless, the optimal solution of the dual problem always leads to good primal solutions [20].

\section{B. Distributed User Association}

Compared to the centralized ones, the distributed UA algorithm has low complexity without demanding the centralized controller. As such, we propose a distributed UA algorithm based on the previous analysis in Section III-A. In the proposed algorithm, each user adopts Algorithm 1 to feedback the UA request to the desired BS, and each BS adopts Algorithm 2 to broadcast the dual variable $\mu_{i}$. Compared to the $\mathcal{O}\left(|\mathcal{B}|^{|\mathcal{U}|}\right)$ complexity of the conventional centralized brute force algorithm, the complexity of the proposed algorithm is $\mathcal{O}(|\mathcal{B}||\mathcal{U}|)$. In addition, the exchanged information between users and BSs is $(|\mathcal{B}|+|\mathcal{U}|)$, compared to the $(|\mathcal{B}| \times|\mathcal{U}|)$ in the centralized algorithm.

\footnotetext{
${ }^{2}$ There are many step size selections such as constant step size and diminishing step size. In this paper, we use the nonsummable diminishing step length, as shown in [19].
}

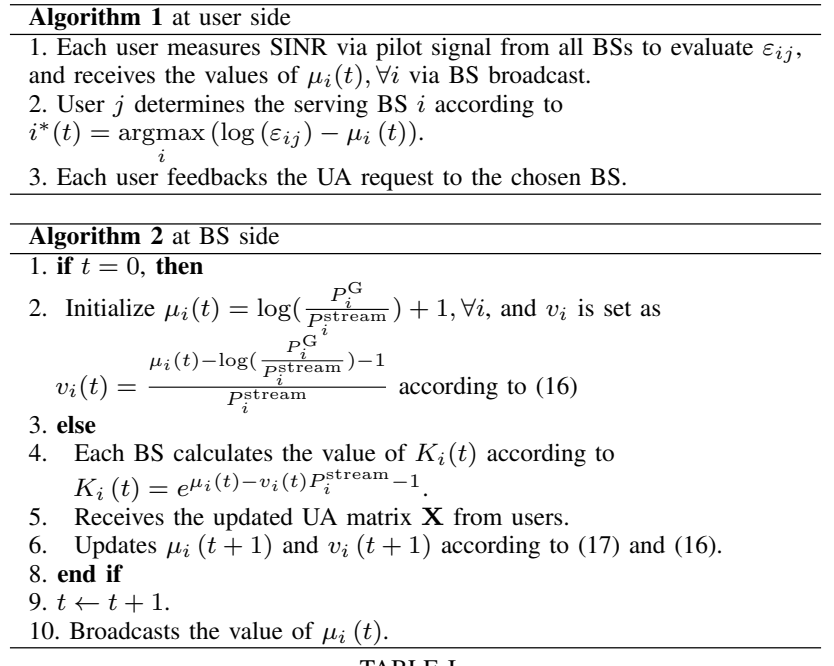

\begin{tabular}{|c|c|}
\hline Parameter & Value \\
\hline Macro cell Bandwidth & $20 \mathrm{MHz}$ \\
\hline $\begin{array}{l}\text { Carrier frequency } \\
\text { of mmWave small cell }\end{array}$ & $28 \mathrm{GHz}$ \\
\hline Inter site distance & $500 \mathrm{~m}$ \\
\hline Thermal Noise power & $-174 \mathrm{dBm} / \mathrm{Hz}$ \\
\hline Path loss of MBS & $15.3+37.6 \log _{10} d(\mathrm{~m})$ \\
\hline Path loss of mmWave BS & $\begin{array}{c}\alpha+10 \beta \log _{10} d(m)+\xi \\
\xi \sim \mathcal{N}\left(0, \sigma^{2}\right) \\
\text { LOS }: \alpha=61.4, \beta=2 \\
\sigma=5.8 \mathrm{~dB} ; \mathrm{NLOS}: \alpha=72.0 \\
\beta=2.92, \sigma=8.7 \mathrm{~dB}[14]\end{array}$ \\
\hline $\begin{array}{l}\text { Probability of Outage(O)-LOS-NLOS } \\
\text { in mmWave small cell }\end{array}$ & $\begin{array}{c}\text { O: } P_{\mathrm{o}}(d)=\max \left\{0,1-e^{-\frac{d}{30}+5.2}\right\} \\
\text { LOS: } p_{\mathrm{L}}(d)=\left(1-P_{\mathrm{o}}(d)\right) e^{-\frac{d}{67.1}} \\
\quad \text { NLOS: } 1-P_{\mathrm{o}}(d)-p_{\mathrm{L}}(d)\end{array}$ \\
\hline Min harvested power of MBS & $32 \mathrm{dBm}$ \\
\hline Max harvested power of MBS & $58 \mathrm{dBm}$ \\
\hline Min harvested power of mmWave BS & $22 \mathrm{dBm}$ \\
\hline Max harvested power of mmWave BS & $48 \mathrm{dBm}$ \\
\hline $\begin{array}{l}\text { Transmit power of MBS } \\
\text { per user stream }\end{array}$ & $30 \mathrm{dBm}$ \\
\hline $\begin{array}{l}\text { Transmit power of mmWave BS } \\
\text { per user stream }\end{array}$ & $25 \mathrm{dBm}$ \\
\hline Power consumption of phase shifter & $19.5 \mathrm{~mW}[17]$ \\
\hline Efficiency of Power amplifier & 0.3 \\
\hline
\end{tabular}
TABLE I

Simulation PARAMETERS

\section{SIMULATION RESULTS}

In this section, we present numerical results to evaluate the performance of the proposed UA algorithm and the impacts of massive MIMO and mmWave. We consider a downlink HetNet composed of 19 macro cells. In each macro cell, mmWave small cell BSs are randomly deployed and 30 users are uniformly distributed. The coefficients under ZF precoding for power consumption are $\Lambda_{0,0}=4, \Lambda_{1,0}=$ $4.8, \Lambda_{2,0}=0, \Lambda_{3,0}=2.08 * 10^{-8}, \Lambda_{0,1}=1, \Lambda_{1,1}=$ $9.5 * 10^{-8}$, and $\Lambda_{2,1}=6.25 * 10^{-8}$ [15]. The basic simulation parameters are as defined in Table I. Then we compare the performance of the proposed UA algorithm with the max RSRP scheme and max SINR scheme.

First we set the number of mmWave BS antennas and the number of mmWave BSs in each macro cell as 100 
and 5 , respectively. Fig. 2 demonstrates the throughput of each user versus number of MBS antennas for different $S$. The mmWave BW is $1 \mathrm{GHz}$. We can see that regardless of the number of MBS antennas and the maximum number of data stream $\mathrm{S}$, the proposed UA algorithm achieves higher throughput than the other two UA schemes. For the same S, the throughput of the proposed UA algorithm increases with the number of MBS antennas, while the throughput of the other two schemes decrease. This can be explained by twofold: 1) For max RSRP UA and max SINR UA, the load of mmWave small cells decreases by increasing the number of MBS antennas, since users are more likely to be associated with the MBSs that provide larger antenna gains; and 2) Given the harvested power, adding more MBS antennas increases the power consumption of the MBS, which decreases the amount of users that MBS can supply. It also demonstrates that under the same number of MBS antennas, the relatively larger S achieves better throughput performance.

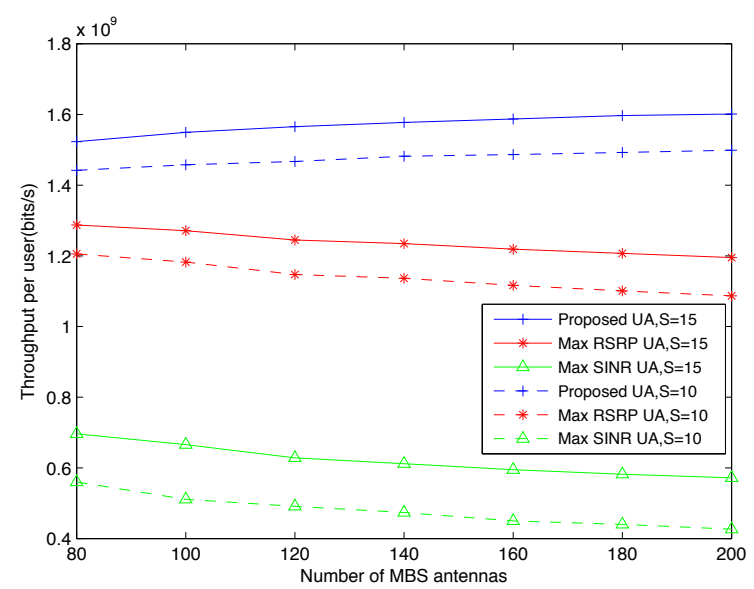

Fig. 2. Throughput per user versus number of MBS antennas for different S.

Then we set the number of MBS antennas as 100 and $\mathrm{S}$ as 10, respectively. Fig. 3 shows the throughput per user versus number of mmWave BS antennas for different mmWave BWs. It can be seen that under the same BW, the proposed algorithm outperforms the other two schemes. When the antenna number of mmWave small cells is larger, the throughput is higher for all three UA schemes due to the fact that the mmWave antenna gain increases and more users are offloaded to the mmWave small cells. For the proposed UA and max RSRP UA, the increase of mmWave BW brings about a significant increase in the throughput, which indicates the advantage of mmWave transmission. However, the use of max SINR UA does not experience a large increase in throughput as mmWave BW increases, since the load of the mmWave small cell is light compared to the other two UA algorithms.

Fig. 4 shows the throughput per user versus number of mmWave small cells in each macro cell. We set the number of MBS antennas as 100 and $S=10$. The mmWave BW is 1

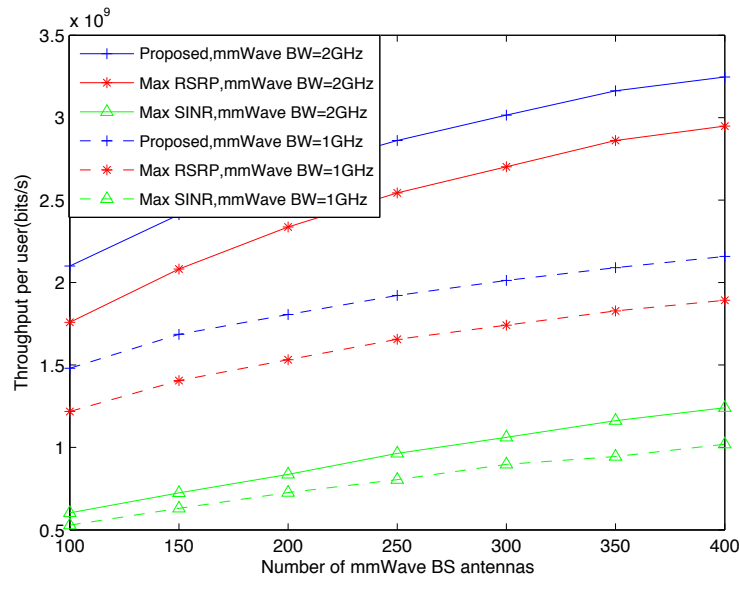

Fig. 3. Throughput per user versus number of mmWave BS antennas for different mmWave BWs.

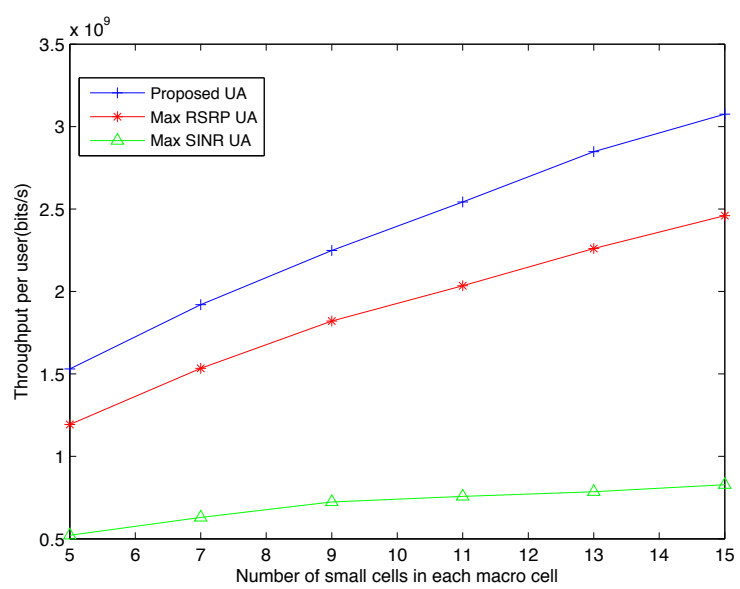

Fig. 4. Throughput per user versus number of mmWave small cells in each macro cell.

GHz. It is seen that the proposed UA scheme obtains higher throughput than the max RSRP UA and max SINR UA. For the proposed UA and max RSRP UA, deploying more mmWave small cells can significantly increases the throughput, since the distance between the user and mmWave BS becomes shorter and more users are offloaded to the mmWave small cells with larger BWs. An interesting phenomenon is seen that for max SINR UA, as the number of mmWave BSs exceeds a critical value (approximately 9 in this figure), the throughput slightly increases. The reason is that the interference of mmWave tier increases with the number of mmWave small cells, which results in a slow increase in SINR and thus a slow increase in the load of mmWave small cells.

Fig. 5 demonstrates the percentage of throughput contributed by massive MIMO MBS and mmWave small cells. We assume that the number of MBS antennas and mmWave 


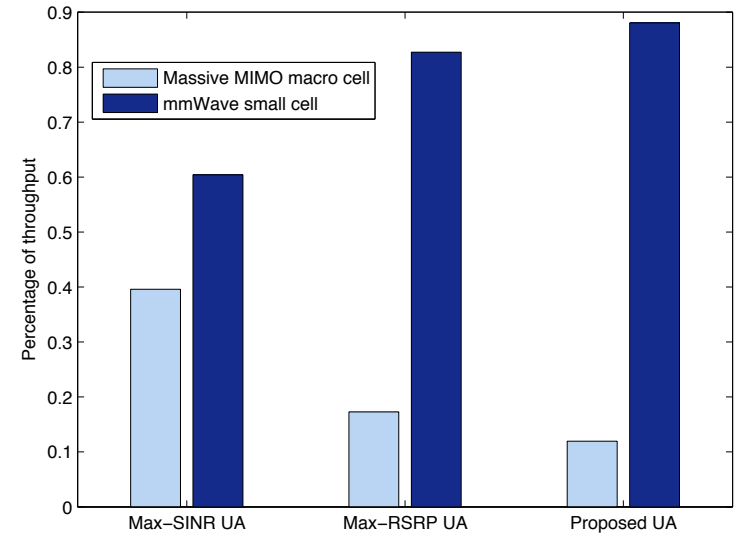

Fig. 5. Percentage of throughput occupied by the first tier consisting of massive MIMO macro cells and the second tier consisting of mmWave small cells.

BS antennas are the same as $100, \mathrm{~S}=10$, the mmWave $\mathrm{BW}$ is $1 \mathrm{GHz}$, and there are five mmWave small cells in each macro cell. We see that among the three UA algorithms, mmWave small cells perform the highest percentage of throughput by using the proposed UA algorithm, and the lowest percentage of throughput by using max SINR UA algorithm, which indicates that the superiority of the proposed UA algorithm results from offloading more users to the mmWave small cells.

\section{CONCLUSIONS}

In this paper, we took into account a two-tier hybrid heterogeneous network consisting of massive MIMO macro cells and millimeter wave small cells. All the base stations were powered by the harvested energy from the renewable energy sources. These emerging techniques pose great challenges to the user association designs. As such, we utilized a proportionally fair network utility optimization approach and proposed a novel distributed user association algorithm. Simulation results showed that the proposed algorithm performs better than the two typical user association schemes, namely max SINR and max RSRP user association algorithms. In contrast to these two schemes, the throughput will not decrease with the increasing number of MBS antennas by using the proposed algorithm. Increasing the number of mmWave base stations, mmWave base station antennas or mmWave bandwidths brings significant improvement of the throughput. In addition, we demonstrated that mmWave small cells have larger contribution to the throughput in hybrid HetNets than massive MIMO.

\section{REFERENCES}

[1] 5G-PPP 5G Vision. [Online]. Available: https://5g-ppp.eu/wpcontent/uploads/2015/02/5G-Vision-Brochure-v1.pdf

[2] J. G. Andrews, S. Buzzi, W. Choi, S. V. Hanly, A. Lozano, A. C. K. Soong, and J. C. Zhang, "What will 5G be?" IEEE J. Sel. Areas Commun., vol. 32, no. 6, pp. 1065-1082, Jun. 2014.

[3] E. Hossain and M. Hasan, "5G cellular: Key enabling technologies and research challenges," IEEE Instrumentation Measurement Mag., vol. 18, no. 3, pp. 11-21, Jun. 2015.

[4] H. Q. Ngo, E. G. Larsson, and T. L. Marzetta, "Energy and spectral efficiency of very large multiuser MIMO systems," IEEE Trans. Commun., vol. 61, no. 4, pp. 1436-1449, Apr. 2013.

[5] Z. Pi and F. Khan, "An introduction to millimeter-wave mobile broadband systems," IEEE Commun. Mag., vol. 49, no. 6, pp. 101-107, June 2011.

[6] IEEE 802.11-10/0433r2, PHY/MAC Complete Proposal Specification (TGad D0.1), 2010.

[7] V. Jungnickel, K. Manolakis, W. Zirwas, B. Panzner, V. Braun, M. Lossow, M. Sternad, R. Apelfrojd, and T. Svensson, "The role of small cells, coordinated multipoint, and massive MIMO in 5G," IEEE Commun. Mag., vol. 52, no. 5, pp. 44-51, May 2014.

[8] H. S. Ghadikolaei, C. Fischione, G. Fodor, P. Popovski, and M. Zorzi, "Millimeter wave cellular networks: A MAC layer perspective," IEEE Trans. Commun., pp. 1-22, 2015.

[9] D. Bethanabhotla, O. Bursalioglu, H. Papadopoulos, and G. Caire, "User association and load balancing for cellular massive MIMO," in Information Theory and Applications Workshop (ITA), 2014, Feb. 2014, pp. $1-10$.

[10] G. Athanasiou, P. Weeraddana, C. Fischione, and L. Tassiulas, "Optimizing client association for load balancing and fairness in millimeter-wave wireless networks," IEEE/ACM Trans. Netw., vol. 23, no. 3, pp. 836850, June 2015

[11] M. Zheng, P. Pawelczak, S. Stanczak, and H. Yu, "Planning of cellular networks enhanced by energy harvesting," IEEE Commun. Lett., vol. 17 , no. 6, pp. 1092-1095, June 2013.

[12] S. Singh, M. N. Kulkarni, A. Ghosh, and J. G. Andrews, "Tractable model for rate in self-backhauled millimeter wave cellular networks," arXiv preprint arXiv:1407.5537, July 2014.

[13] K. Venugopal, M. C. Valenti, and R. W. Heath, Jr., "Interference in finitesized highly dense millimeter wave networks," in Proc. Information Theory and Applications (ITA), San Diego, CA, Feb. 2015.

[14] M. R. Akdeniz, Y. Liu, M. K. Samimi, S. Sun, S. Rangan, T. S. Rappaport, and E. Erkip, "Millimeter wave channel modeling and cellular capacity evaluation," IEEE J. Sel. Areas Commun., vol. 32, no. 6 , pp. 1164-1179, June 2014.

[15] E. Björnson, L. Sanguinetti, J. Hoydis, and M. Debbah, "Designing multi-user MIMO for energy efficiency: When is massive MIMO the answer?" in Proc. 2014 IEEE WCNC, Apr. 2014.

[16] S. Rangan, T. Rappaport, E. Erki, Z. Latinovic, M. R. Akdeniz, and Y. Liu, "Energy efficient methods for millimeter wave picocellular systems," in IEEE Communication Theory Workshop, 2013.

[17] O. Orhan, E. Erkip, and S. Rangan, "Low power analog-to-digital conversion in millimeter wave systems: Impact of resolution and bandwidth on performance," in Proc. Information Theory and Applications (ITA), San Diego, CA, Feb. 2015.

[18] A. Garcia-Saavedra, P. Serrano, A. Banchs, and M. Hollick, "Energyefficient fair channel access for IEEE 802.11 WLANs," in Proc. 2011 IEEE Int. Symp. on a World of Wireless, Mobile and Multimedia Networks (WoWMoM), Jun. 2011, pp. 1-9.

[19] S. Boyd and A. Mutapcic, Subgradient methods. Stanford University, 2008.

[20] K. Shen and W. Yu, "Distributed pricing-based user association for downlink heterogeneous cellular networks," IEEE J. Sel. Area in Commun., vol. 32, no. 6, pp. 1100-1113, Jun. 2014. 